\title{
TOPOLOGICAL SPACES WITH PRESCRIBED NONCONSTANT CONTINUOUS MAPPINGS ${ }^{1}$
}

BY

\author{
VĚRA TRNKOVÁ
}

\begin{abstract}
Given a $T_{1}$-space $Y$ and a $T_{3}$-space $V$, consider $T_{3}$-spaces $X$ such that $X$ has a closed covering by spaces homeomorphic to $V$ and any continuous mapping $f: X \rightarrow Y$ is constant. All such spaces and all their continuous mappings are shown to form a very comprehensive category, containing, e.g., a proper class of spaces without nonconstant, nonidentical mappings or containing a space $X$, for every monoid $M$, such that all the nonconstant continuous mappings of $X$ into itself are closed under composition and form a monoid isomorphic to $M$. The category of paracompact connected spaces, having a closed covering by a given totally disconnected paracompact space, has, e.g., analogous properties. Categories of metrizable spaces are also investigated.
\end{abstract}

I. Introduction. Let us begin with the well-known result of de Groot [3] that every group is isomorphic to the group of all homeomorphisms of a topological space onto itself. In 1964, at the Colloquium on Topology in Tihany, he posed the problem whether any monoid (i.e., a semigroup with unit element) is isomorphic to the monoid of all nonconstant continuous mappings of a topological space into itself. Let us note that the set of all nonconstant continuous mappings does not always form a monoid; the composition of two nonconstant mappings can be constant. The exact formulation is as follows. Given a monoid $M$, does there exist a space $X$ such that the set of all nonconstant continuous mappings of $X$ onto itself is closed under composition and this set, endowed with this composition, forms a monoid isomorphic to $M$ ? The first step towards the solution of this problem was given by $Z$. Hedrlín in [4]. He constructed a reflexive binary relation $R$ on a set $X$ such that all the nonconstant $R$-preserving mappings of $X$ into itself form a monoid isomorphic to a given monoid $M$. The relation $R$ induces a closure space on $X$ by the rule

$$
\operatorname{cl} A=\{x \in X \mid(a, x) \in R \text { for some } a \in A\},
$$

and $R$-preserving mappings are just continuous mappings of this closure space. The full solution of the above problem is given in [14]; there, every monoid $M$ is represented by all the nonconstant continuous mappings of a metric space into itself. In [15], every monoid is also represented by all the nonconstant continuous mappings of a compact Hausdorff space into itself (this was already proved in [14] but only under the assumption that there is no measurable cardinal). In all these

Received by the editors April 26, 1978 and, in revised form, October 24, 1978.

AMS (MOS) subject classifications (1970). Primary 54C05, 54H10, 54H15, $18 \mathrm{~B} 15$.

${ }^{1}$ The contents of this paper have been presented to the IVth Prague Topological Symposium in August 1976. 
papers, a more general setting, namely full and almost full embeddings of categories, is considered.

Let us recall some usual notions about categories and functors. A functor $\Phi$ : $K \rightarrow H$ is called $a$ full embedding if it is an isofunctor of $K$ onto a full subcategory of $H$. A category $U$ is called universal if every concrete category (i.e., a category admitting a faithful functor into the category of all sets and all mappings) can be fully embedded in it. $U$ is called $s$-universal if every small category (i.e., a category whose objects form a set) can be fully embedded in it.

The investigation of full embeddings of categories was started by J. R. Isbell in [9], and then developed by Z. Hedrlín, A. Pultr, L. Kučera and others in a number of papers. Let us recall several results needed in the present paper. We denote by $\mathbf{G}$ the category of all connected directed graphs and all compatible mappings (for the exact definition, see §IV of the present paper). In [5], $\mathbf{G}$ is proved to be $s$-universal. In [11], $G$ is proved to be universal under the following set-theoretical assumption:

(M) Relatively measurable cardinals are not cofinal in the class of all cardinals.

Let $\mathbf{P}$ be the category whose objects are all pairs $(S, \mathcal{T})$, where $S$ is a nonempty set, and $\mathcal{T}$ is a nonempty subset of $\exp S ; f:(S, \mathcal{T}) \rightarrow\left(S^{\prime}, \mathcal{T}^{\prime}\right)$ is a morphism of $\mathbf{P}$ iff it is a mapping of $S$ into $S^{\prime}$ such that, for every $T$ in $\sigma^{\prime}, f^{-1}(T)$ is in $\mathcal{T}$. Thus, $\mathbf{P}$ is the category of all "topological spaces without axioms" and all their continuous mappings. In [11], L. Kučera proved that $P$ is universal (without any set-theoretical assumption). Let us mention that these and many other facts about full embeddings are systematically presented in the monograph [13].

Now, let Top be the category of all topological spaces and all their continuous mappings, let $T$ be one of its full subcategories. A functor $\Phi: K \rightarrow T$ is called an almost full embedding if, for any pair $a, b$ of objects of $K$,

$$
f: a \rightarrow b \stackrel{\Phi}{\leftrightarrow} \Phi(f): \Phi(a) \rightarrow \Phi(b)
$$

is a bijection of the set of all $K$-morphisms of $a$ into $b$ onto the set of all nonconstant continuous mappings of $\Phi(a)$ into $\Phi(b)$, i.e., $\Phi$ is "full up to constant mappings". $T$ is called almost universal (or almost $s$-universal) if every concrete category (or every small category, respectively) can be almost fully embedded on it. Clearly,

if $K$ is universal (or $s$-universal) and $\Phi: K \rightarrow T$ is an almost full embedding, then $T$ is almost universal (or almost $s$-universal, respectively).

What is really presented in [14] is a construction of an almost full embedding $\Re$ of $\mathbf{G}$ into the category $\mathbf{M}$ of all metrizable spaces; hence $\mathbf{M}$ is almost $s$-universal (and, under (M), universal). Since every monoid $M$ can be considered as a category with precisely one object, it can be fully embedded into $\mathbf{G}$, i.e. there exists a connected graph $G$ such that the monoid of all compatible mappings of $G$ into itself is isomorphic to $M$. Then the metrizable space $\mathscr{R}(G)$ gives the representation of $M$ by all nonconstant continuous mappings, i.e. the solution of the above problem of de Groot. 
Almost full embeddings of universal or $s$-universal categories are also used for construction of stiff classes of spaces. Let us recall that a class $\mathbf{C}$ of topological spaces is called stiff if, for every $X, Y \in \mathbf{C}$ and every continuous mapping $f$ : $X \rightarrow Y$, either $f$ is constant or $X=Y$ and $f$ is the identity (sometimes, the words rigid or strongly rigid are also used; the investigation of stiff classes of spaces was also started by de Groot in [3]). Let a cardinal $\alpha$ be given, let $k(\alpha)$ be a discrete category (i.e., with no morphisms except the identities) such that its objects form a set of cardinality $\alpha$. Since $k(\alpha)$ is a small category, it can be almost fully embedded into every almost $s$-universal category. Its image is a stiff set (of cardinality $\alpha$ ) of spaces. Hence, by [14], there are arbitrarily large stiff sets of metrizable spaces. Since "large discrete category" (i.e., a discrete category whose objects form a proper class) is concrete, every almost universal category contains a stiff proper class of spaces. Hence, under (M), there is a stiff proper class of metrizable spaces. In [10], V. Koubek constructed an almost full embedding of the universal category $\mathbf{P}^{\text {op }}$, opposite to $\mathbf{P}$, into the category of paracompact spaces. Hence there is a stiff proper class of paracompact spaces (without any set-theoretical requirement).

As a final remark of the introduction, let us note that the proof of the universality of $\mathbf{P}$ is deep and difficult. On the other hand, we give here quite a simple proof that $\mathbf{P}$ contains a proper rigid class, i.e. a proper class of objects without nonidentical morphisms. Hence, the existence of stiff proper classes of spaces, based on almost full embeddings of $\mathbf{P}$ into full subcategories of Top, can be easily verified.

Proposition. P contains a proper rigid class.

Proof. For every infinite cardinal $\alpha$ put $\mathscr{T}(\alpha)=\mathscr{T}_{1}(\alpha) \cup \mathcal{T}_{2}(\alpha)$, where

$$
\mathcal{T}_{1}(\alpha)=\{\{x, y\} \mid x, y \in \alpha\}, \quad \mathscr{T}_{2}(\alpha)=\{\{z \in \alpha \mid z \geqslant x\} \mid x \in \alpha\} .
$$

(As usual, every cardinal is considered as the set of all smaller ordinals.) We show that if $f:(\alpha, \mathcal{T}(\alpha)) \rightarrow(\beta, \mathcal{T}(\beta))$ is a morphism of $\mathbf{P}$, then necessarily $\alpha=\beta$ and $f$ is the identity. Since $\{x\}$ is in $\mathcal{T}(\beta)$ for every $x \in \beta$ and $\varnothing$ is not in $\mathcal{T}(\alpha), f$ must be a mapping onto $\beta$. Let us suppose $f(x)=f(y)$ for some $x, y \in \alpha, x \neq y$. Choose $z \in \beta$ such that $f(0) \neq z$ and $f(x) \in p=\{f(0), z\}$. Since $p$ is in $\mathcal{T}(\beta), f^{-1}(p)$ is in $\mathcal{T}(\alpha)$. Since card $f^{-1}(p) \geqslant 3, f^{-1}(p)$ must be in $\mathcal{T}_{2}(\alpha)$. Since $f^{-1}(p)$ contains 0 , it must be equal to the whole $\alpha$, which is a contradiction. We conclude that $\alpha=\beta$ and $f$ is a bijection. Then $f^{-1}(T) \in \mathcal{T}_{2}(\alpha)$ whenever $T \in \mathcal{T}_{2}(\beta)$. This implies, by transfinite induction, that $f$ is the identity.

II. The main theorems. Let us add two aspects to the field of problems mentioned in the introduction.

(A) All the results described in the introduction, say that there are spaces such that all nonconstant continuous mappings between any pair of them have some prescribed properties. A classical question of topology is about nonconstant continuous mappings into a given space. Let us recall the regular space without nonconstant continuous real functions of E. Hewitt [8] and J. Novák [12] and the 
following well-known generalization of $H$. Herrlich [6]. For any $T_{1}$-space $Y$ there exists a regular space $X$ with more than one point and such that any continuous mapping $f: X \rightarrow Y$ is constant. Now, we can ask about the coherence of these problems. For example, let a $T_{1}$-space, $Y$, and a monoid, $M$, be given. Does there exist a space $X$ (regular, if possible) such that any continuous mapping $f: X \rightarrow Y$ is constant and all nonconstant continuous mappings of $X$ into itself form a monoid isomorphic to $M$ ? We give much more than merely the affirmative answer to this question.

(B) Embeddings of spaces into spaces with prescribed properties are often met with in topology and can also be investigated in the above field of problems. For example, given a space $V$ and a monoid $M$, does there exist a space $X$ containing $V$ (nicely embedded, if possible; for example, as a closed subspace) such that all nonconstant continuous mappings of $X$ into itself form a monoid isomorphic to $M$ ? For $T_{1}$-spaces $V$, we prove a stronger result than the affirmative answer to this question.

In what follows, all topological spaces are supposed to be $T_{1}$-spaces. Speaking about categories of topological spaces, we always mean these spaces and all their continuous mappings, i.e. the full subcategories of Top.

Let $V$ be a topological space. We say that a space $X$ contains $V$ many times if for any $v \in V$ and any $x \in X$ there exists a homeomorphism $h$ of $V$ onto a closed subspace of $X$ such that $h(v)=x$.

The aim of the present paper is to prove the following theorems.

THEOREM 1. Let a space $Y$ be given. Let $V$ be a space (Hausdorff or regular). Then all the spaces (Hausdorff spaces or regular spaces, respectively) $X$ containing $V$ many times and such that any continuous mapping $f: X \rightarrow Y$ is constant, form an almost universal category.

THEOREM 2. Let $V$ be a completely regular (normal or paracompact) totally disconnected space. Then all completely regular (normal or paracompact) connected spaces, containing $V$ many times, form an almost universal category.

TheOREM 3. Let $V$ be a metrizable totally disconnected space. Then all connected metrizable spaces, containing $V$ many times, form an almost $s$-universal category and, under (M), they form an almost universal category.

In fact, we prove a more general statement, depending not only on the given spaces $Y$ and $V$ but also on some "parameters" (it is formulated in $\S \mathrm{V}$.14) and by suitable choices of parameters, we obtain all the above theorems.

\section{Basic construction.}

1. Let $P$ be a topological space with a distinguished point $p$. Let $Q$ be a topological space with three distinct distinguished points, say $q_{1}, q_{2}, q_{3}$. With respect to these data, we define a space $X$ (denoted by $(P, p) *\left(Q, q_{1}, q_{2}, q_{3}\right)$, if it is necessary to indicate the given data), by induction, as follows:

$$
X_{0}=P, \quad o_{0}=p .
$$


If $X_{n}$ and its distinguished point $o_{n}$ are defined, we put

$$
Y_{n+1}=X_{n} \vee \underset{y \in X_{n} \backslash\left\{o_{n}\right\}}{\bigvee}(Q \times\{y\}),
$$

where $\bigvee$ denotes the sum (= coproduct) in the category Top, i.e. the disjoint union as clopen subspaces, $X_{n+1}$ is the quotient space of $Y_{n+1}$ obtained by the identifications

$$
\begin{gathered}
\left(q_{1}, y\right) \sim y \text { and }\left(q_{2}, y\right) \sim o_{n} \text { for any } y \in X_{n} \backslash\left\{o_{n}\right\}, \\
\left(q_{3}, y\right) \sim\left(q_{3}, y^{\prime}\right) \text { for any } y, y^{\prime} \in X_{n} \backslash\left\{o_{n}\right\} .
\end{gathered}
$$

The point obtained by the last identification is defined to be $o_{n+1}$. To simplify the notation, we shall suppose that $X_{n} \subset X_{n+1}, X_{n}$ is a subspace of $X_{n+1}, Q \times\{y\}$ is a subspace of $X_{n+1}$ for any $y \in X_{n} \backslash\left\{o_{n}\right\}$ and

$$
\left(q_{1}, y\right)=y, \quad\left(q_{2}, y\right)=o_{n}, \quad\left(q_{3}, y\right)=o_{n+1}
$$

for any $y \in X_{n} \backslash\left\{o_{n}\right\}$. Put $X=\cup_{n=0}^{\infty} X_{n}$ with the inductively generated topology on $X$.

OBSERVATION. The sequence $\left\{o_{n}\right\}$ has no accumulation point in $X$.

PROPOSITION. If $P$ and $Q$ both are Hausdorff (or regular or completely regular or normal or paracompact), then $X$ has the same property.

The routine proof is omitted.

2. The metric form of the construction. Let $P, Q$ be metric spaces, let us suppose that $\operatorname{diam} P \leqslant 1, \operatorname{diam} Q \leqslant 1$, $\operatorname{dist}\left(q_{i}, q_{j}\right)=1$ whenever $i \neq j$. We define $X_{0}=P$, $o_{0}=p$ again and

$$
Y_{n+1}=X_{n} \vee \underset{y \in X_{n} \backslash\left\{o_{n}\right\}}{\bigvee}(Q \times\{y\})
$$

again, but now $\vee$ denotes the sum (= coproduct) in the category Metr of all metric spaces with diameter $\leqslant 1$ and all their contractions (we recall that $f$ is a contraction if

$$
\operatorname{dist}(f(x), f(y)) \leqslant \operatorname{dist}(x, y))
$$

always, i.e., the summands are embedded into $Y_{n+1}$ isometrically and the distance of points of distinct summands is equal to $1 . X_{n+1}$ is a quotient space of $Y_{n+1}$ in the category Metr, obtained by the same identifications

$$
\begin{gathered}
\left(q_{1}, y\right) \sim y, \quad\left(q_{2}, y\right) \sim \sigma_{n} \text { for all } y \in X_{n} \backslash\left\{o_{n}\right\}, \\
\left(q_{3}, y\right) \sim\left(q_{3}, y^{\prime}\right) \quad \text { for all } y, y^{\prime} \in X_{n} \backslash\left\{o_{n}\right\} ;
\end{gathered}
$$

the point obtained is $o_{n+1}$. In more detail, if $\tau$ is the metric of $Y_{n+1}$, then $\sigma(x, y)$ is defined as inf $\sum_{i=0}^{k} \tau\left(a_{i}, b_{i}\right)$, where the sum is taken over a chain $\left(a_{0}, b_{0}, a_{1}, b_{1}, \ldots, a_{k}, b_{k}\right)$ such that $x=a_{0}, b_{0} \sim a_{1}, b_{1} \sim a_{2}, \ldots, b_{k}=y$, and the infimum is taken over all these chains.

OBSERVATION. $\sigma$ is really a metric on $X_{n+1}, X_{n}$ is embedded into $X_{n+1}$ isometrically.

Hence, we may suppose $X_{n} \subset X_{n+1}$ as a metric space. Consequently, there is the unique metric on $X=\cup_{n=0}^{\infty} X_{n}$ such that all the $X_{n}$ are subspaces. 
ObSERVATION. $\operatorname{diam} X=1, \operatorname{dist}\left(o_{n}, o_{m}\right)=1$ whenever $n \neq m . X$ is complete whenever $P$ and $Q$ are complete.

In what follows, we consider the topological case, i.e. the construction in Top, as well as the metric case, i.e. the construction in Metr. The text is written for Top, differences for the metric case are sketched in parentheses, if necessary.

3. For any $y \in X_{n} \backslash\left\{o_{n}\right\}$, denote by $e_{y}: Q \rightarrow X$ the mapping sending $q \in Q$ to $(q, y)$. Clearly, $e_{y}$ is a homeomorphism into (and a contraction in the metric case). For every $y \in X$ denote by $\lambda(y)$ the smallest natural number such that $y \in$ $X_{n} \backslash\left\{o_{n}\right\}$.

OBSERVATION. If $e_{y}\left(q_{2}\right)=e_{y^{\prime}}\left(q_{2}\right)$ or $e_{y}\left(q_{3}\right)=e_{y^{\prime}}\left(q_{3}\right)$, then $\lambda(y)=\lambda\left(y^{\prime}\right)$. If, moreover, $e_{y}\left(q_{1}\right)=e_{y^{\prime}}\left(q_{1}\right)$, then $y=y^{\prime}$.

4. For every $l$ define

$$
\rho_{l+1, l}: X_{l+1} \cup\left\{o_{m} \mid m>l\right\} \rightarrow X, \cup\left\{o_{m} \mid m>l\right\}
$$

by

$$
\begin{gathered}
\rho_{l+1, l}(z)=z \quad \text { whenever } z \in X_{l} \cup\left\{o_{m} \mid m>l\right\}, \\
\rho_{l+1, l}(q, x)=x \quad \text { whenever } x \in X \backslash\left\{o_{l}\right\}, \quad q \in Q \backslash\left\{q_{1}, q_{2}, q_{3}\right\} .
\end{gathered}
$$

Clearly, $\rho_{l+1, l}$ is continuous at every point $y \in X_{l+1} \backslash\left\{o_{l}, o_{l+1}\right\}$. Now, we define a space $\tilde{X}_{l}$ as $\left(X_{l} \backslash\left\{o_{l}\right\}\right) \cup\left\{o_{m} \mid m \geqslant l\right\}$, where the topology on $X_{l} \backslash\left\{o_{l}\right\}$ is as in $X$ and all the points $o_{m}$, including the point $o_{l}$, are isolated in $\tilde{X}_{l}$. Define

$$
\tilde{\rho}_{l}: X \backslash\left\{o_{m} \mid m \geqslant l\right\} \rightarrow \tilde{X}_{l}
$$

as follows. Put $\tilde{\rho}_{l}(z)=z$ whenever $\lambda(z) \leqslant l$. If $n=\lambda(z)>l$, consider the mapping $\rho=\rho_{n, n-1} \circ \cdots \circ \rho_{l+1, l}$ and put $\tilde{\rho}_{l}(z)=\rho(z)$.

OBSERVATION. $\tilde{\rho}_{l}$ is a continuous mapping.

Hence, for every $n$, a domain-range-restriction of $\tilde{\rho}_{n+1}$ defines a continuous retraction of $\left(X_{n+1} \backslash\left\{o_{n+1}\right\}\right) \cup \cup{ }_{x \in X_{n}} C_{x}$, where $C_{x}$ is the component of $X \backslash\left\{o_{m} \mid m>n\right\}$ containing $x$, onto $X_{n+1} \backslash\left\{o_{n+1}\right\}$.

Define

$$
\rho_{l}: X \backslash\left\{o_{m} \mid m \geqslant l\right\} \stackrel{\tilde{\rho}_{l}}{\rightarrow} \tilde{X}_{l} \rightarrow X,
$$

where the last arrow is the inclusion.

ObSERVATION. If $C$ is a connected subset of $X \backslash\left\{o_{m} \mid m=0,1,2, \ldots\right\}$ and $\rho_{0}$ maps it onto $o_{n}$, then $\rho_{n}$ also maps it onto $o_{n}$.

5. Let a space $V$ be given. Let $W$ be the quotient space of $\bigvee_{v \in V}(V \times\{v\})$ obtained by the identifications $(v, v) \sim\left(v^{\prime}, v^{\prime}\right)$ for all $v, v^{\prime} \in V$, the point obtained by these identifications is denoted by $w$. (For the metric case, we suppose diam $V \leqslant 1$ and the construction proceeds in Metr.)

ObSERVATION. If $W$ is a closed subspace of $Q$ and $q_{1}=w$, then $X$ contains $V$ many times.

6. Given a space $W$ with its distinguished point $w$, we define a $W$-tree $T$ as follows: $T_{0}=W, T_{n+1}$ is the quotient space of $T_{n} \vee \bigvee_{t \in T_{n}}(W \times\{t\})$ given by the identifications $t \sim(w, t)$ for all $t \in T_{n}$. We suppose $T_{n} \subset T_{n+1}$ and $T$ is 
defined as $\cup_{n=0}^{\infty} T_{n}$ with the inductively generated topology. (For the metric case, we suppose diam $W \leqslant 1$ and the construction proceeds in Metr.) $w$ is called the root of the tree $T$.

7. Let $W$ be a space with a distinguished point $w$. Let $Q$ contain $W$ as a closed subspace such that $w=q_{1}$ and $q_{2}, q_{3} \in Q \backslash W$. Define a binary relation $\stackrel{\circ}{\sim}$ on $X=(P, p) *\left(Q, q_{1}, q_{2}, q_{3}\right)$ as follows. The relation $\stackrel{\circ}{\sim}$ is the equality on $X_{0}$, i.e., $x \stackrel{\circ}{\sim}$ implies $x=y$ whenever $x, y \in X_{0}$. If $\stackrel{\circ}{\sim}$ is already defined on $X_{n}$, we extend it onto $X_{n+1}$ by $x \stackrel{\circ}{\sim} y$ iff either $x=y$ or $x \in W \times\{\bar{x}\}, y \in W \times\{\bar{y}\}$ and $\bar{x} \stackrel{\circ}{\sim} \overline{\text {. }}$ This definition really extends the relation $\stackrel{\circ}{\sim}$ because $o_{n} \stackrel{\circ}{\sim}$ for no $z \in X_{n} \backslash\left\{o_{n}\right\}$; on the other hand, if $\bar{x}, \bar{y} \in X_{n} \backslash\left\{o_{n}\right\}$, then $\bar{x}=\left(q_{1}, \bar{x}\right), \bar{y}=\left(q_{1}, \bar{y}\right)$ and $q_{1}=w \in$ $W$.

ObSERvation. The relation $\dot{\sim}$ is an equivalence on $X$. It defines a closed decomposition of $X$ into subsets homeomorphic to the $W$-tree $T$. We call them $W$-branches of $X$. A point $x$ of $X$ is a root of its $W$-branch (i.e., the $W$-branch which contains it) if either $x \in X_{0}$ or $x \in e_{y}(Q \backslash W)$ for some $y \in X$. If $m \neq n$, then $o_{n}$ and $o_{m}$ are contained in distinct $W$-branches.

8. Let nonempty spaces $V$ and $Y$ be given. Construct the space $W$ and its distinguished point $w$ as in 5. Let $H$ be a space and $h_{1}, h_{2}$ two of its distinct points such that no continuous mapping of $H$, either into $Y$ or into the $W$-tree $T$, distinguishes $h_{1}$ from $h_{2}$. Denote by $Z$ the quotient of $H \vee W$, where we identify $h_{1}$ and $w$. (For the metric case, we suppose $\operatorname{diam} V \leqslant 1, \operatorname{diam} H \leqslant 1, \operatorname{dist}\left(h_{1}, h_{2}\right)=1$ and the sums and the quotients go in Metr.) In what follows, we work with a space $Q$

(a) containing $Z$ as a closed subspace;

(b) $q_{1}=h_{1}=w, q_{2}=h_{2} \in Q \backslash W$,

(c) $q_{3} \in Q \backslash Z$.

ObSERvation. For any $(P, p)$, the space $X=(P, p) *\left(Q, q_{1}, q_{2}, q_{3}\right)$ has the following properties:

$(\alpha)$ It contains $V$ many times.

( $\beta$ ) Any continuous mapping of $X$ into $Y$ or into the $W$-tree is constant.

( $\gamma$ ) The equivalence $\stackrel{\circ}{\sim}$ described in 7 defines a closed decomposition of $X$ into $W$-branches.

9. Let $P$ and $P^{\prime}$ be spaces with distinguished points $p$ and $p^{\prime}$. Let $Q$ and $q_{1}, q_{2}, q_{3}$ be as in 8. Put $X=(P, p) *\left(Q, q_{1}, q_{2}, q_{3}\right), X^{\prime}=\left(P^{\prime}, p^{\prime}\right) *\left(Q, q_{1}, q_{2}, q_{3}\right)$. We use the notation in the construction of $X$ as in 1 and the same notation for $X^{\prime}$, the symbols being primed only, i.e. $X^{\prime}=\cup_{n=0}^{\infty} X_{n}^{\prime}, e_{z}^{\prime}: Q \rightarrow X^{\prime}$ sends $q$ to $(q, z)$ for $z \in X^{\prime}$ and so on.

Let $\mathcal{F}$ be the set of all continuous mappings $f: P \rightarrow P^{\prime}$ such that $f(p)=p^{\prime}$, $f(P \backslash\{p\}) \subset P^{\prime} \backslash\left\{p^{\prime}\right\}$. For every $f \in \mathcal{F}$ define $\bar{f}: X \rightarrow X^{\prime}$ as follows: $\bar{f}(x)=f(x)$ for all $x \in X_{0}$; if $\bar{f}$ is defined on $X_{n}$, extend it on $X_{n+1}$ by $\bar{f}(q, y)=(q, \bar{f}(y))$ for every $q \in Q, y \in X_{n} \backslash\left\{o_{n}\right\}$. One can see easily that $e_{y} \circ \bar{f}=e_{\bar{f}(y)}^{\prime}$; particularly, $\bar{f}\left(o_{n}\right)=o_{n}^{\prime}$ for all $n$.

10. $Q$ and $q_{1}, q_{2}, q_{3}$ are supposed to be as in 8 ; the other notation as in 9 . 
LeMma A. Let every continuous mapping $k: Q \rightarrow X^{\prime}$ either be equal to $e_{z}^{\prime}$ for some $z \in X^{\prime}$ or map the whole $Q$ into one $W$-branch of $X^{\prime}$. Let $g: X \rightarrow X^{\prime}$ be a continuous mapping. Then either $g$ is constant or $e_{y} \circ g=e_{g(y)}^{\prime}$ for all $y \in X$.

Proof. By our assumption, $e_{y} \circ g$ either is equal to $e_{z}^{\prime}$ for some $z \in X^{\prime}$ (and then necessarily $z=g(y)$ ) or maps $Q$ into one $W$-branch. Hence, we have only to prove that if there exists $y \in X$ such that $e_{y} \circ g$ maps $Q$ into one $W$-branch, then $g$ is constant. Denote $n=\lambda(y)$ (for the definition of the symbol $\lambda$ see 3 ). Then $e_{y}\left(q_{2}\right)=o_{n}, e_{y}\left(q_{3}\right)=o_{n+1}$. Since $o_{l}^{\prime}$ and $o_{l+1}^{\prime}$ are in distinct $W$-branches of $X^{\prime}$ for every $l$, no $e_{b} \circ g$ can be equal to any $e_{z}^{\prime}$ whenever $b \in X_{n} \backslash\left\{o_{n}\right\}$. Consequently $g$ maps the whole $X_{n+1}$ into one $W$-branch. Then, for every $y \in X_{n+1} \backslash\left\{o_{n+1}\right\}, g$ maps $e_{y}(Q)$ into one $W$-branch because $g(y)=g\left(e_{y}\left(q_{1}\right)\right)$ and $g\left(o_{n+1}\right)$ are contained in one $W$-branch. Now, we can proceed by induction. We conclude that $g$ maps the whole $X$ into one $W$-branch of $X^{\prime}$. Then $g$ is constant, by $8(\beta)$.

LEMMA B. Let the following two conditions be fulfilled.

(i) Every continuous mapping $Q \rightarrow X^{\prime}$ either is equal to some $e_{z}^{\prime}$ or it maps the whole $Q$ into one $W$-branch of $X^{\prime}$.

(ii) Every continuous mapping $P \rightarrow X^{\prime}$ maps $P$ either into $P^{\prime}$ or into one $W$-branch of $X^{\prime}$.

Then any continuous mapping $g: X \rightarrow X^{\prime}$ is either constant or equal to $\bar{f}$ for some $f \in \mathcal{F}$.

Proof. By Lemma A, either $g$ is constant or $e_{y} \circ g=e_{g(y)}^{\prime}$ for all $y \in X$. We have to prove that, in the second case, $g=\bar{f}$ for some $f \in \mathscr{F}$. If $g$ maps $P$ into one $W$-branch of $X^{\prime}$, then for any $y \in P \backslash\{p\}$, the equation $e_{y} \circ g=e_{g(y)}^{\prime}$ cannot be true. We conclude that $g$ maps $P$ into $P^{\prime}$, by (ii) and $g(p)=p^{\prime}, g(y) \neq p^{\prime}$ whenever $y \in P \backslash\{p\}$. Consequently, the domain-range restriction $f: P \rightarrow P^{\prime}$ of $g$ is in $\mathscr{F}$. Clearly, $g=\bar{f}$.

IV. The triangle-construction and the basic functors. Let $\mathbf{P}$ be the category defined in $\S \mathbf{I}$. Let $\mathbf{G}$ be the category of all connected directed graphs, mentioned in $\S \mathbf{I}$, i.e. objects are all pairs $(S, R)$, where $S$ is a nonempty set, $R \subset S \times S$ and

for every $s_{1}, s_{2} \in S$ (not necessarily distinct) there exist $t_{1}=s_{1}$, $t_{2}, \ldots, t_{n}=s_{2}$ in $S$ such that either $\left(t_{i}, t_{i+1}\right)$ or $\left(t_{i+1}, t_{i}\right)$ is in $R$;

$f:(S, R) \rightarrow\left(S^{\prime}, R^{\prime}\right)$ is a morphism of $\mathbf{G}$ iff it is a mapping of $S$ into $S^{\prime}$ such that $\left(f\left(s_{1}\right), f\left(s_{2}\right)\right) \in R^{\prime}$ whenever $\left(s_{1}, s_{2}\right) \in R$. We recall that $\mathbf{G}$ is $s$-universal and, under (M), it is universal by [5] and [11]; $P$ is universal by [11]. In [14], an almost full embedding $\Re$ of $\mathbf{G}$ into Top is constructed such that any $\mathscr{T}(S, R)$ is metrizable (in fact, a complete metric semicontinuum). In [10], an almost full embedding $\mathscr{P}$ : $\mathbf{P}^{\text {op }} \rightarrow$ Top is constructed, where $\mathbf{P}^{\text {op }}$ is the category opposite to $\mathbf{P}$, such that every space $\mathcal{P}(S, \mathscr{T})$ is paracompact. Here, we sketch a modified construction of almost full embeddings $\mathfrak{T}: \mathbf{G} \rightarrow$ Top, $\mathscr{P}: \mathbf{P}^{\text {op }} \rightarrow$ Top, which will be used in the next section. First, we present the triangle construction used in the construction of $\mathfrak{N}$ and $\mathscr{P}$ and also in the next section. 
1. Let $\mathcal{C}$ be a Cook continuum, i.e. a compact metrizable connected space such that

for every subcontinuum $A$ of $\mathcal{C}$ and every continuous mapping $f$ :

$A \rightarrow \mathcal{C}$, either $f$ is constant or $f(x)=x$ for all $x \in A$.

Such a continuum was constructed by $\mathrm{H}$. Cook in [1]. For a more detailed description of the construction, see also [13].

2. The triangle construction. Let $D_{1}, \ldots, D_{4}$ be the following sets of pairs.

$$
\begin{aligned}
D_{1}= & \{(1,2),(2,3),(3,1)\}, \\
D_{2}= & \{(1,(1,1,1)),(2,(1,1,2)),(3,(1,1,3)), \\
& (1,(1,2,3)),(2,(1,2,1)),(3,(1,2,2))\}, \\
D_{3}= & \{((n, 1, j),(n, 2, j)) \mid j=1,2,3, n=1,2, \ldots\}, \\
D_{4}= & \{((n, k, j),(n+1, k, j)) \mid j=1,2,3, k=1,2, n=1,2, \ldots\} .
\end{aligned}
$$

Put $D=D_{1} \cup D_{2} \cup D_{3} \cup D_{4}$ and for any $d=\left(d_{1}, d_{2}\right) \in D$ denote $\pi_{1}(d)=d_{1}$, $\pi_{2}(d)=d_{2}$.

Let $\mathbb{Q}=\left\{A_{d} \mid d \in D\right\}$ be a pairwise disjoint collection of nondegenerate subcontinua of $\mathcal{C}$. We metrize every $A_{d}$ such that if $d \in D_{1}$ then $\operatorname{diam} A_{d}=2^{-1}$, if $d \in D_{2}$ then $\operatorname{diam} A_{d}=2^{-2}$, if $d \in D_{3}, d=((n, 1, j),(n, 2, j))$ then $\operatorname{diam} A_{d}=$ $2^{-(n+1)}$, if $d \in D_{4}, d=((n, k, j),(n+1, k, j))$, then diam $A_{d}=2^{-(n+2)}$. In any $A_{d}$ choose two points $a_{d}^{1}, a_{d}^{2}$ such that $\operatorname{dist}\left(a_{d}^{1}, a_{d}^{2}\right)=\operatorname{diam} A_{d}$. Let $\tilde{A}$ be the quotient of the sum $\bigvee_{a \in D} A_{d}$, both the quotient and the sum considered in the category Metr, given by the identifications

$$
a_{d}^{i} \sim a_{d^{\prime}}^{i^{\prime}}, \quad \text { whenever } \pi_{i}(d)=\pi_{i^{\prime}}\left(d^{\prime}\right) .
$$

Let $A$ be the completion of the space $\tilde{A}$. It is obtained by adding three distinct points to $A$, say $a^{1}, a^{2}, a^{3}, a^{j}$ just being the limit of the sequence $\left\{a_{r_{n}}^{1}\right\}_{n=1}^{\infty}$ with $r_{n}=((n, 1, j),(n+1,1, j))$. The situation is illustrated in the figure. We have $\operatorname{diam} A=1, \operatorname{dist}\left(a^{j}, a^{j^{\prime}}\right)=1$ for $j \neq j^{\prime}$. To simplify the notion, let us suppose that $A_{d} \subset A$. Thus $A_{d} \cap A_{d^{\prime}} \neq \varnothing$ iff $\pi_{k}(d)=\pi_{k^{\prime}}\left(d^{\prime}\right)$ for some $k, k^{\prime} \in\{1,2\}$. If this intersection is nonempty, it consists just of $a_{d}^{k}=a_{d^{\prime}}^{k^{\prime}}$.

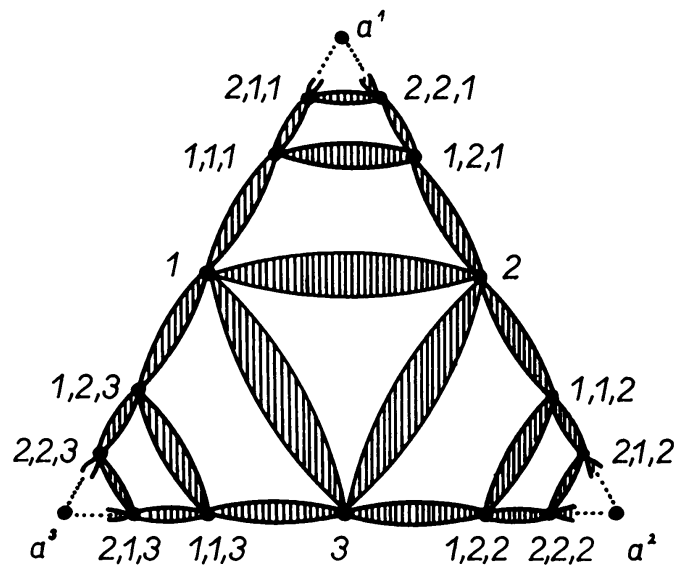


A circle in $A$ is a finite set $\left\{B_{1}, \ldots, B_{n}\right\}$ of distinct members of $\mathbb{Q}$ such that $B_{j} \cap B_{j+1} \neq \varnothing$ for $j=1, \ldots, n-1, B_{n} \cap B_{1} \neq \varnothing$ and $B_{k} \cap B_{l}=\varnothing$ otherwise.

ObSERVATION. For every distinct $d, d^{\prime} \in D, A_{d}$ and $A_{d^{\prime}}$ can be inserted into a circle. More precisely, there exists a circle $\left\{B_{1}, \ldots, B_{n}\right\}$ such that $B_{1}=A_{d}$, $B_{k}=A_{d^{\prime}}$ for $1<k \leqslant n$.

3. First, we construct the almost full embedding $\mathfrak{T}: \mathbf{G} \rightarrow$ Top. Given a connected graph $G=(S, R)$, denote by $\mathscr{N}(S, R)$ the quotient of the sum

$$
\bigvee_{r \in R}(A \times\{r\})
$$

(the sum and the quotient both are taken in Metr) given by the identifications

$$
\begin{array}{ll}
\left(a^{3}, r\right) \sim\left(a^{3}, r^{\prime}\right) & \text { for all } r, r^{\prime} \in R, \\
\left(a^{j}, r\right) \sim\left(a^{j^{\prime}}, r^{\prime}\right) & \text { whenever } \pi_{j}(r)=\pi_{j^{\prime}}\left(r^{\prime}\right), j, j^{\prime} \in\{1,2\}
\end{array}
$$

(where we denote $\pi_{1}\left(s_{1}, s_{2}\right)=s_{1}, \pi_{2}\left(s_{1}, s_{2}\right)=s_{2}$ again). Denote by $\varepsilon_{r}: A \rightarrow \mathfrak{R}(G)$ the embedding of $A$ onto its $r$ th copy, i.e. sending any $a \in A$ to $(a, r)$. The point $\varepsilon_{r}\left(a^{3}\right)$ is denoted by $p_{G}$.

If $f:(S, R) \rightarrow\left(S^{\prime}, R^{\prime}\right)$ is a morphism of $\mathbf{G}$, we define $\Re(f)=g: \operatorname{Tl}(S, R) \rightarrow$ TR $\left(S^{\prime}, R^{\prime}\right)$ by $g\left(a,\left(s_{1}, s_{2}\right)\right)=\left(a,\left(f\left(s_{1}\right), f\left(s_{2}\right)\right)\right)$, i.e.

$$
\varepsilon_{\left(s_{1}, s_{2}\right)} \circ g=\varepsilon^{\prime}\left(f\left(s_{1}\right) f\left(s_{2}\right)\right)
$$

for every $\left(s_{1}, s_{2}\right) \in R$ (where $\varepsilon_{t}^{\prime}$ denotes the embedding of $A$ onto its $t$ th copy in भ( $\left.\left(S^{\prime}, R^{\prime}\right)\right)$.

Clearly, $\mathfrak{T}: \mathbf{G} \rightarrow$ Top is really a functor, any $\mathfrak{T}(S, R)$ is a metrizable space (since the construction is made in $\operatorname{Metr}, \mathfrak{R}(S, R)$ is obtained as a metric space with a complete metric and any $\mathscr{R}(f)$ is a contraction) and

$$
\text { every } \Re(f) \text { maps } p_{G} \text { to } p_{G^{\prime}} \text { and } \mathscr{R}(G) \backslash\left\{p_{G}\right\} \text { into } \mathscr{R}\left(G^{\prime}\right) \backslash\left\{p_{G^{\prime}}\right\} \text {. }
$$

We have only to show that $\mathfrak{N}$ is almost full. This is proved in the three lemmas below.

Lemma A. Let $d_{0}$ be in $D$, let $h: A_{d_{0}} \rightarrow \mathfrak{N}(S, R)$ be a continuous mapping. Then either $h$ is constant or is the domain-restriction of $\varepsilon_{r}: A \rightarrow \mathfrak{N}(S, R)$ for some $r \in R$.

Proof. For every $d \in D, r \in R$, put $\mathscr{Q}_{d, r}=\varepsilon_{r}\left(A_{d} \backslash\left\{a_{d}^{1}, a_{d}^{2}\right\}\right), G_{d, r}=h^{-1}\left(\mathscr{U}_{d, r}\right)$. Let us suppose that $G_{d, r} \neq \varnothing$ for some $r \in R$ and some $d \in D \backslash\left\{d_{0}\right\}$. $G_{d, r}$ is open in $A_{d_{0}}$ and if also $A_{d_{0}} \backslash G_{d, r} \neq \varnothing$, then, by the well-known Kuratowski theorem, the closure of any component of $G_{d, r}$ intersects its boundary. The closure of one such component is a subcontinuum of $A_{d_{0}}$, hence that of the Cook continuum $C$, mapped by $h$ onto a nondegenerate subcontinuum of $\varepsilon_{r}\left(A_{d}\right)$, homeomorphic to a subcontinuum of $\mathcal{C}$, disjoint with $A_{d_{0}}$. This is impossible, hence $A_{d_{0}} \backslash G_{d, r}=\varnothing$, i.e. $h$ maps $A_{d_{0}}$ into $\varepsilon_{r}\left(A_{d}\right)$. Then, necessarily, $h$ is constant. We conclude that if $h$ is nonconstant, then $h\left(A_{d_{0}}\right) \subset \Re(S, R) \backslash \mathcal{U}$, where $\mathscr{U}$ is the union of all $\mathcal{U}_{d, r}$ with $r \in R, d \in D \backslash\left\{d_{0}\right\}$. Since $h\left(A_{d_{0}}\right)$ is connected, nondegenerate, it is contained in $\varepsilon_{r}\left(A_{d_{0}}\right)$ for some $r \in R$. Then necessarily $h$ is the domain-restriction of $\varepsilon_{r}$.

Lemma B. Let $h: A \rightarrow \mathfrak{T}(S, R)$ be a continuous mapping. Then either $h$ is constant or $h=\varepsilon_{r}$ for some $r \in R$. 
Proof. By Lemma A, $h$ maps every $A_{d}$ either onto one point or onto $\varepsilon_{r(d)}\left(A_{d}\right)$ like the $\varepsilon_{r(d)}$. Let $\left\{B_{1}, \ldots, B_{n}\right\}$ be a circle in $A$. If $h$ maps $B_{1}$ onto one point, then necessarily it maps all the $B_{i}$ onto the point. Since any $A_{d}, A_{d^{\prime}}$ with $d \neq d^{\prime}$ can be inserted into a circle in $A, h$ is constant whenever it maps some $A_{d}$ onto one point. Otherwise, the $r(d)$ 's are all equal to some $r \in R$ and $h=\varepsilon_{r}$.

LemMa C. Let $g: \Re(S, R) \rightarrow \operatorname{T}\left(S^{\prime}, R^{\prime}\right)$ be a continuous mapping. Then either $g$ is constant or $g=\mathfrak{T}(f)$ for a morphism $f:(S, R) \rightarrow\left(S^{\prime}, R^{\prime}\right)$ of $\mathbf{G}$.

Proof. For every $r \in R, \varepsilon_{r} \circ g$ is either constant or equal to $\varepsilon_{r^{\prime}}^{\prime}$ for some $r^{\prime} \in R^{\prime}$, by Lemma B. Since $(S, R)$ is supposed to be connected, $g$ is constant whenever $\varepsilon_{r} \circ g$ is constant for some $r \in R$. Otherwise $\varepsilon_{r} \circ g=\varepsilon_{r^{\prime}}^{\prime}$ for every $r \in R$. In this second case, for every $s \in S$ choose $r \in R$ such that $\pi_{j}(r)=s$ for $j \in\{1,2\}$ and put $f(s)=\pi_{j}\left(r^{\prime}\right)$. Then $f:(S, R) \rightarrow\left(S^{\prime}, R^{\prime}\right)$ is a morphism of $\mathbf{G}$ and $g=\mathscr{N}(f)$.

4. Now, we describe the construction of $\mathcal{P}: \mathbf{P}^{\text {op }} \rightarrow$ Top. Let $D, \mathbb{Q}=\left\{A_{d} \mid d \in D\right\}$ and $A$ be as in 2, let $B$ be a nondegenerate subcontinuum of $\mathcal{C}$, disjoint with all the $A_{d}$. Choose three distinct points in $B$, say $b, 0,1$. For every object $\omega=(S, \mathcal{T})$ of $\mathbf{P}$, denote by $B^{S}$ the space of all mappings of $S$ into $B$, endowed with the topology of pointwise convergence, by $b^{S}$ the constant mapping of $S$ onto $b$ and, for every $T \in \mathcal{T}$, by $\chi_{T}: S \rightarrow B$ the point of $B^{S}$ with $\chi_{T}(s)=1$ if $s \in T, \chi_{T}(s)=0$ if $s \notin T$. Then $\mathscr{P}(\omega)$ is defined as the quotient space of $B^{S} \vee \bigvee_{T \in \mathscr{F}}(A \times\{T\})$ given by the identifications

$$
\begin{gathered}
\left(a^{1}, T\right) \sim \chi_{T}, \quad\left(a^{2}, T\right) \sim b^{S} \quad \text { for every } T \in \mathcal{T}, \\
\left(a^{3}, T\right) \sim\left(a^{3}, T^{\prime}\right) \quad \text { for every } T, T^{\prime} \in \mathcal{T} .
\end{gathered}
$$

The point obtained by the last identification is denoted by $p_{\omega}$. Since $A$ and $B$ are compact and metrizable, $\mathscr{P}(\omega)$ can be easily shown to be paracompact.

Now, let $f: \omega \rightarrow \omega^{\prime}$ be a morphism of $\mathbf{P}, \omega=(S, \mathcal{T}), \omega^{\prime}=\left(S^{\prime}, \mathcal{T}^{\prime}\right) . \mathscr{P}(f)=g$ : $\mathscr{P}\left(\omega^{\prime}\right) \rightarrow \mathscr{P}(\omega)$ is defined as follows. It sends any $\varphi: S^{\prime} \rightarrow B$ to $f \circ \varphi: S \rightarrow B$ and any $(a, T) \in A \times\{T\}$ to $\left(a, f^{-1}(T)\right) \in A \times\left\{f^{-1}(T)\right\}$ (this is correct because $T \in \mathcal{T}^{\prime}$ implies $\left.f^{-1}(T) \in \mathcal{T}\right)$. One can verify that this definition is compatible with the above identifications, i.e. if $x \sim y$ then $g(x) \sim g(y)$, consequently $g$ is a correctly defined continuous mapping. Clearly, $\mathcal{P}: \mathbf{P}^{\text {op }} \rightarrow$ Top is really a functor and, for any $f: \omega \rightarrow \omega^{\prime}$ in $\mathbf{P}, \mathcal{P}(f)$ maps $p_{\omega^{\prime}}$ to $p_{\omega}$ and $\mathscr{P}\left(\omega^{\prime}\right) \backslash\left\{p_{\omega^{\prime}}\right\}$ into $\mathscr{P}(\omega) \backslash\left\{p_{\omega}\right\}$. It remains to show that $\mathscr{P}$ is almost full. We prove it in the three lemmas below.

Lemma A. Let $h: A \rightarrow \mathcal{P}(\omega)$ be a continuous mapping. Then either $h$ is constant or $h(a)=(a, T)$ for some $T \in \mathcal{T}$ and all $a \in A$.

Proof. (a) First, we prove that $h$ maps every $A_{d}$ either onto one point or $h(a)=(a, T(d))$ for some $T(d) \in \mathcal{T}$ and all $a \in A_{d}$. For every $d \in D, T \in \mathcal{T}$, denote $\mathscr{U}_{d, T}=\left(A_{d} \backslash\left\{a_{1}^{d}, a_{2}^{d}\right\}\right) \times\{T\}$. Put $C=\{0,1\}^{S} \subset B^{S}$ and $\mathscr{U}_{0}=$ $B^{S} \backslash\left(C \cup\left\{b^{s}\right\}\right)$. If $d \in D$ is given, put $\mathscr{U}_{d}=\left\{\mathscr{U}_{0}\right\} \cup\left\{\mathscr{U}_{d^{\prime}, T} \mid T \in \mathcal{T}, d^{\prime} \in\right.$ $D \backslash\{d\}\}$. Every $\mathcal{U} \in \mathcal{U}_{d}$ is open in $\mathcal{P}(\omega)$, hence $G=h^{-1}(\mathcal{Q}) \cap A_{d}$ is open in $A_{d}$. If $G \neq \varnothing$ and $A_{d} \backslash G \neq \varnothing$, then we obtain the contradiction with the Kuratowski theorem as in the proof of Lemma $\mathrm{A}$ in 3. Hence, if $G \neq \varnothing$, then necessarily $h\left(A_{d}\right) \subset$ Q , i.e., $h$ is constant on $A_{d}$. If $h$ is not constant on $A_{d}$, we obtain that 


$$
h\left(A_{d}\right) \subset \mathscr{P}(\omega) \backslash \bigcup_{\mathcal{U} \in \mathscr{U d}} \mathscr{U} .
$$

Since $h\left(A_{d}\right)$ is connected, nondegenerate (and $C$ is totally disconnected) there exists $T(d) \in \mathcal{T}$ such that $h(a)=(a, T(d))$ for all $a \in A_{d}$.

(b) If $h$ is constant on some $A_{d}$, then it is constant. The proof of this is quite analogous as the proof of Lemma B in 3. Otherwise, $h(a)=(a, T(d))$ for some $T(d) \in \mathcal{T}$ and all $a \in A$. Then necessarily all the $T(d)$ 's are equal to each other.

Lemma B. Let $S^{\prime}$ be a set, $h: B^{S^{\prime}} \rightarrow \mathcal{P}(\omega)$ be a continuous mapping. Then either $h$ is constant or $h\left(B^{S^{\prime}}\right) \subset B^{S}$.

Proof. First, let us notice that every continuous mapping of $B$ into $\mathscr{P}(\omega)$ is either constant or maps $B$ into $B^{S}$. The proof of this is quite analogous to part (a) of the previous proof. Now, let $S^{\prime}$ be a nonempty set such that $h\left(\varphi_{0}\right) \notin B^{S}$ for some $\varphi_{0} \in B^{S^{\prime}}$. For any $t \in S^{\prime}$ define $\eta_{t}: B \rightarrow B^{S^{\prime}}$ such that $\eta(x)=\varphi$ where $\varphi(s)=\varphi_{0}(s)$ for all $s \in S^{\prime} \backslash\{t\}, \varphi(t)=x$. Then $\eta_{t} \circ h$ is constant. Consequently $h(\varphi)=h\left(\varphi_{0}\right)$ whenever $\varphi$ differs from $\varphi_{0}$ for at most one $s \in S^{\prime}$. By induction, $h(\varphi)=h\left(\varphi_{0}\right)$ whenever $\varphi$ differs from $\varphi_{0}$ for a finite number of $s \in S^{\prime}$. These $\varphi^{\prime} s$ form a dense subset of $B^{S^{\prime}}$, hence $h$ maps $B^{S^{\prime}}$ onto $h\left(\varphi_{0}\right)$.

LEMMA C. Let $g: \mathcal{P}\left(\omega^{\prime}\right) \rightarrow \mathscr{P}(\omega)$ be a continuous mapping. Then either $g$ is constant or $g=\mathscr{P}(f)$ for a morphism $f: \omega \rightarrow \omega^{\prime}$ of $\mathbf{P}$.

Proof. (a) First, let us suppose that $g$ is constant on $B^{S^{\prime}}$. Then $g\left(b^{S^{\prime}}\right)=g\left(\chi_{T}\right)$ for every $T \in \mathcal{T}^{\prime}$; hence $g$ is constant on every $A \times\{T\}$, by Lemma A. Then $g$ is constant.

(b) Let $g$ be nonconstant on $B^{S^{\prime}}$. Then $g\left(B^{S^{\prime}}\right) \subset B^{S}$, by Lemma B. For any $s \in S$, denote by $\pi_{s}: B^{S} \rightarrow B$ the $s$ th projection. By [7], $g \circ \pi_{s}: B^{S^{\prime}} \rightarrow B$ is either constant or a projection. Let us suppose that $g$ is constant on $A \times\{T\}$ for some $T \in \mathcal{T}^{\prime}$. Then, for every $s \in S, \pi_{s}\left(g\left(b^{S^{\prime}}\right)\right)=\pi_{s}\left(g\left(\chi_{T}\right)\right)$. Since every projection $B^{S^{\prime}} \rightarrow B$ maps $b^{S^{\prime}}$ to $b$ and $\chi_{T}$ in $\{0,1\}, g \circ \pi_{s}$ cannot be equal to a projection, hence it is constant. But then $g$ is constant on $B^{S^{\prime}}$, which is a contradiction. We conclude that $g$ is nonconstant on any $A \times\{T\}$; it maps it onto $A \times\{\tilde{T}\}$ by the rule $(a, T) \leadsto(a, \tilde{T})$, by Lemma A. Moreover, for every $s \in S, g \circ \pi_{s}$ is a projection, say an $f(s)$ th one. Consequently, $f: S \rightarrow S^{\prime}$ is a mapping such that $g(\varphi)=$ $f \circ \varphi$ for every $\varphi \in B^{S^{\prime}}$. One can verify that $\tilde{T}=f^{-1}(T)$, i.e., $f:(S, \mathcal{T}) \rightarrow\left(S^{\prime}, \mathcal{T}\right)$ is a morphism of $\mathbf{P}$ and $g=\mathscr{P}(f)$.

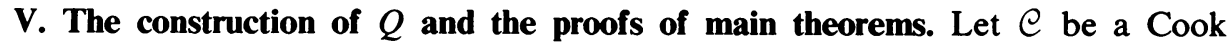
continuum; let $D$ be as in $\S I V .2$. Let $B$ be a nondegenerate subcontinuum of $C$; let $\mathbb{Q}^{i}=\left\{A_{d}^{i} \mid d \in D\right\}, i \in\{1,2,3\}$, be collections of nondegenerate subcontinua of $\mathcal{C}$ such that

$$
\mathcal{E}=\{B\} \cup \bigcup_{i=1}^{3} \mathbb{Q}^{i}
$$


is pairwise disjoint. Let $\mathfrak{T}: \mathbf{G} \rightarrow$ Top be the almost full embedding constructed as in $\$ I V .3$ by means of $\mathbb{Q}^{3}$ (the upper index 3 is added to the symbols in the construction, if necessary) and let $\mathscr{P}: \mathbf{P}^{\text {op }} \rightarrow$ Top be the almost full embedding constructed as in $\S \mathrm{IV} .4$ by means of $B$ and $\mathbb{Q}^{3}$.

In this section, we construct $Q$ and $q_{1}, q_{2}, q_{3}$ by means of $\mathbb{Q}^{1}$ and $\mathbb{Q}^{2}$ such that if $(P, p)=\left(\mathscr{N}(G), p_{G}\right)$ or $(P, p)=\left(\mathscr{P}(\omega), p_{\omega}\right)$, where $\mathfrak{N}$ and $\mathscr{P}$ are constructed by means of $\mathbb{Q}^{3}$ (or $B$ and $\mathbb{Q}^{3}$, respectively), then Lemma $B$ from $\$$ III.10 can be applied. Then the functors

$$
\Phi: \mathbf{G} \rightarrow \text { Top, } \quad \Psi: \mathbf{P}^{\text {op }} \rightarrow \text { Top }
$$

defined by

$$
\begin{array}{cc}
\Phi(G)=\left(\mathscr{T}(G), p_{G}\right) *\left(Q, q_{1}, q_{2}, q_{3}\right), & \Phi(f)=\overline{\mathscr{T}(f)} \\
\Psi(\omega)=\left(\mathscr{P}(\omega), p_{\omega}\right) *\left(Q, q_{1}, q_{2}, q_{3}\right), & \Psi(f)=\overline{\mathscr{P}(f),}
\end{array}
$$

(for the definition of the strip, see §III.9) are almost full embeddings of $s$-universal category $\mathbf{G}$ and universal category $\mathbf{P}^{\circ \mathbf{p}}$.

1. Let spaces $V$ and $Y$ be given. Construct $W$ and its distinguished point $w$ as in §III.5. Let $T$ be a $W$-tree (for the definition, see $\$$ III.6). Let $\mathcal{E}$ be as above, i.e. a pairwise disjoint collection of nondegenerate subcontinua of a Cook continuum $C$, $\mathcal{E}$ has the form $\{B\} \cup \cup{ }_{i=1}^{3} \mathbb{Q}^{i}$. Let $H$ be a space and $h_{1}, h_{2}$ its two distinct points such that

(1) no continuous mapping of $H$ into $Y$ or into $T$ distinguishes $h_{1}$ from $h_{2}$;

(2) every continuous mapping of any subcontinuum of every $E \in \mathcal{E}$ into $H$ is constant.

(Let us note that (1) has been already required in III.8.) We construct the space $Q$ and its points $q_{1}, q_{2}, q_{3}$ in the dependence of these data. Suitable choices of $V, Y$, $\mathcal{E}, H$, fulfilling (1) and (2), and leading to the proofs of the main theorems, will be shown in $\S \mathrm{V} .14$.

(For the metric form of the construction, we require diam $V \leqslant 1$, diam $Y \leqslant 1$, $\operatorname{diam} H=1, \operatorname{dist}\left(h_{1}, h_{2}\right)=1$.)

2. Let $V, Y, W, w, \mathcal{E}, H, h_{1}, h_{2}$ be as in 1, let (1) and (2) be fulfilled. Let $Z$ be as in $\S$ III.8, i.e. the quotient of the sum $H \vee W$, given by the identification $w \sim h_{1}$. We denote this point $q_{1}$ and put $h_{2}=q_{2}$.

Let $M$ be a connected rigid relation on the underlying set of the space $Z$ (we denote it also by $Z)$, i.e. $(Z, M)$ is an object of $\mathbf{G}$ such that if $f:(Z, M) \rightarrow(Z, M)$ is a morphism of $\mathbf{G}$, then $f$ is the identity. Such a relation has been constructed in [17]. Denote by $\pi_{1}: M \rightarrow Z, \pi_{2}: M \rightarrow Z$ the first and the second projections. Let $A^{i}$ and its points $a^{i, 1}, a^{i, 2}, a^{i, 3}$ be constructed by means of $\mathbb{Q}^{i}$ as in $\S I V .2(i=1,2,3)$. The space $Q$ is defined as the quotient of the sum (either in Top or in Metr whenever $Z$ is a metric space, $\operatorname{diam} Z \leqslant 1$, $\operatorname{dist}\left(q_{1}, q_{2}\right)=1$ )

$$
Z \vee \underset{z \in Z}{\bigvee}\left(A^{1} \times\{z\}\right) \bigvee \underset{m \in M}{\bigvee}\left(A^{2} \times\{m\}\right)
$$


given by the following identifications

$$
\begin{aligned}
& \left(a^{1,1}, z\right) \sim z \quad \text { for all } z \in Z ; \\
& \left(a^{1,2}, z\right) \sim\left(a^{2, j}, m\right) \quad \text { iff } \pi_{j}(m)=z \\
& \left(a^{1,3}, z\right) \sim\left(a^{2,3}, m\right) \quad \text { for all } z \in Z \text { and all } m \in M .
\end{aligned}
$$

The point obtained by the last identification is defined to be $q_{3}$. To simplify the notation, let us suppose that $Z \subset Q, H$ and $W$ are both subspaces of $Z$ and $H \cap W=\left\{q_{1}\right\}, q_{2} \in H, \xi_{z}: A^{1} \rightarrow Q$ sends $A^{1}$ onto the $z$ th copy $A^{1}$ in $Q$ by the rule $\xi_{z}(a)=(a, z)$, and $\xi_{m}: A^{2} \rightarrow Q$ sends $A^{2}$ onto the $m$ th copy $A^{2}$ in $Q$ by the rule $\xi_{m}(a)=(a, m)$, for every $z \in Z$ and every $m \in M$. Hence, $\xi_{z}\left(a^{1,1}\right)=z, \xi_{z}\left(a^{1,3}\right)=$ $q_{3}=\xi_{m}\left(a^{2,3}\right)$ and $\xi_{z}\left(a^{1,2}\right)=\xi_{m}\left(a^{2, j}\right)$ iff $\pi_{j}(m)=z$.

OBSERVATION. $Q$ is a connected space. It is Hausdorff or regular or normal or paracompact whenever $Z$ has this property. In the metric case, diam $Q=1$ and $\operatorname{dist}\left(q_{i}, q_{j}\right)=1$ for $i \neq j ; Q$ is complete whenever $Z$ is complete.

3. In what follows, either $(P, p)=\left(\mathscr{N}(G), p_{G}\right)$ or $(P, p)=\left(\mathscr{P}(\omega), p_{\omega}\right)$, $\mathfrak{N}$ and $\mathscr{P}$ are constructed by means of $\mathbb{Q}^{3}$ (and $B$ ), and $Q$ and $q_{1}, q_{2}, q_{3}$ are as in 2. Denote $X=(P, p) *\left(Q, q_{1}, q_{2}, q_{3}\right)$. As in $\S I I I .1 .3$, we suppose that $P \subset X$ and, for every $x \in X, e_{x}: Q \rightarrow X$ sends $Q$ onto its $x$ th copy in $X$ by the rule $e_{x}(q)=(q, x)$.

Denote $K_{1}=Z, K_{2}=M$. Denote by $\Gamma$ the set of all $\gamma=(j, k, x)$ with $j \in$ $\{1,2\}, k \in K_{j}, x \in X$ and by $\sigma_{\gamma}$ the embedding $A^{j} \stackrel{\xi_{k}}{\rightarrow} Q \stackrel{e_{x}}{\rightarrow} X$. By our convention (see §IV.2), $A_{d}^{j} \subset A^{j}$ for every $d \in D$.

We recall that $W$-branches of $X$ and their roots have been introduced in §III.7-8.

ObSERVATION. For every $x \in X$ and $z \in Q \backslash W, e_{x}(z)$ is the root of its $W$-branch (see §III.7). Particularly, if $\gamma=(2, k, x)$, then every point of $\sigma_{\gamma}\left(A^{2}\right)$ is the root of its $W$-branch; if $\gamma=(1, k, x)$, then every point of $\sigma_{\gamma}\left(A^{1} \backslash\left\{a^{1,1}\right\}\right)$ is the root of its $W$-branch. We conclude that if for some $\gamma, \gamma^{\prime} \in \Gamma, a \in \sigma_{\gamma}\left(A^{j}\right)$ and $a^{\prime} \in \sigma_{\gamma^{\prime}}\left(A^{j^{\prime}}\right)$ are in the same $W$-branch, then either $\gamma=\gamma^{\prime}, a=a^{\prime}$ or $j=j^{\prime}=1$ and $a=\sigma_{\gamma}\left(a^{1,1}\right)$, $a^{\prime}=\sigma_{\gamma^{\prime}}\left(a^{1,1}\right)$. Particularly, if for some $\gamma, \gamma^{\prime} \in \Gamma, d, d^{\prime} \in D$, some $a \in \sigma_{\gamma}\left(A_{d^{\prime}}^{j^{\prime}}\right)$ and $a^{\prime} \in \sigma_{\gamma^{\prime}}\left(A_{d^{\prime}}^{j^{\prime}}\right)$ are in the same $W$-branch, then necessarily $\gamma=\gamma^{\prime}$ (hence $j=j^{\prime}$ ), $a=a^{\prime}$ and the pairs $d, d^{\prime}$ have a common member, i.e. $\pi_{l}(d)=\pi_{l^{\prime}}\left(d^{\prime}\right)$ for some $l, l^{\prime} \in\{1,2\}$.

4. Lemma. Let $E$ be in $\mathcal{E}$, let $F$ be a subcontinuum of $E$. Let $h: F \rightarrow Q$ be continuous. Then either $h$ is constant or $h(F) \subset W$ or there exist $d \in D, j \in\{1,2\}$, $k \in K_{j}$ such that $E=A_{d}^{j}$ and $h$ is the domain-restriction of $A^{j} \stackrel{\xi_{k}}{\rightarrow} Q$.

Proof. Let $j \in\{1,2\}, d \in D$ and $A_{d}^{j}$ be distinct from $E$. Let $k$ be in $K_{j}$. Put थ $=\xi_{k}\left(A_{d}^{j} \backslash\left\{a_{d}^{j, 1}, a_{d}^{j, 2}\right\}\right), G=h^{-1}(\mathcal{Q})$. If $G \neq \varnothing, F \backslash G \neq \varnothing$, then, by the Kuratowski theorem, the closure of every component of $G$ intersects its boundary; hence $F$ contains a subcontinuum mapped by $h$ onto a nondegenerate subcontinuum of $\xi_{k}\left(A_{d}^{j}\right)$, which is impossible. Thus, if $G \neq \varnothing$, then necessarily $F \backslash G=\varnothing$, i.e., $h$ maps $F$ into $\xi_{k}\left(A_{d}^{j}\right)$, hence it must be constant. We conclude that $h$ is either constant or it maps $F$ into $Q \backslash \cup \xi_{k}\left(A_{d}^{j} \backslash\left\{a_{d}^{j, 1}, a_{d}^{j, 2}\right\}\right)$, where the union is over all 
$(j, d)$ such that $A_{d}^{j}$ is distinct from $E$ and all $k \in K_{j}$. Since $h(F)$ is connected and every continuous mapping of $F$ into $H$ is constant, by (2), either $h(F) \subset W$ or $h$ is constant or $E=A_{d}^{j}$ for some $j \in\{1,2\}, d \in D$ and $h(F) \subset \xi_{k}\left(A_{d}^{j}\right)$ for some $k \in K_{j}$. Then necessarily $h$ is the domain-restriction of $\xi_{k}$.

5. LEMMA. For every $E \in \mathbb{Q}^{1} \cup \mathbb{Q}^{2}$ and every subcontinuum $F$ of $E$, any continuous mapping $h: F \rightarrow P$ is constant.

Proof. Since either $P=\mathscr{T}(G)$ or $P=\mathscr{P}(\omega)$, both are constructed by means of $\mathbb{Q}^{3}$ (and $B$ ). The proof is quite analogous to the proof of Lemma A in $\$ I V .3$ (or the first part of the proof of Lemma $A$ in $\$ I V .4$, respectively).

6. Let $\left\{o_{n}\right\}_{n=0}^{\infty}$ be as in §III.1. By §III.1, this sequence has no accumulation point in $X$. For every $E \in \mathcal{E}$ and any continuous $h: E \rightarrow X, h(E)$ is compact, hence it contains at most a finite number of its members. Let us suppose that for some $E \in \mathcal{E}$ and some continuous mapping $h: E \rightarrow X, h(E)$ really intersects $\left\{o_{n}\right\}_{n=0}^{\infty}$. Let $n$ be the greatest natural number such that $o_{n} \in H(E)$. Since $h(E)$ is connected, the continuous mapping

$$
\rho_{n+1}: X \backslash\left\{o_{m} \mid m>n\right\} \rightarrow \tilde{X}_{n+1}
$$

introduced in $\S$ III.4, defines a continuous retraction of $h(E) \cup\left(X_{n+1} \backslash\left\{o_{n+1}\right\}\right)$ onto $X_{n+1} \backslash\left\{o_{n+1}\right\}$.

LEMMA. $h \circ \rho_{n+1}: E \rightarrow X_{n+1} \backslash\left\{o_{n+1}\right\}$ is a constant with the value $o_{n}$.

Proof. For every $x \in X_{n} \backslash\left\{o_{n}\right\}$, put $\mathscr{U}_{x}=e_{x}\left(Q \backslash W \cup\left\{q_{1}, q_{2}, q_{3}\right\}\right)$. Let $G_{x}$ be its preimage in $h \circ \rho_{n+1}$. Since $o_{n} \in h(E)$, we have $E \backslash G_{x} \neq \varnothing$. If $G_{x} \neq \varnothing$, then $h \circ \rho_{n+1}$ maps the closure of every component of $G_{x}$ onto a nondegenerate subcontinuum of $e_{x}(Q)$. By 4 , this is possible only when $h \circ \rho_{n+1}$ maps it into $e_{x}(W)$ or when $h \circ \rho_{n+1}$ is a restriction of $\xi_{k} \circ e_{x}$ for some $k \in K_{j}$ (and $E=A_{d}^{j}$ for some $d \in D$ ). The last case is impossible because $o_{n}=e_{x}\left(q_{2}\right)$ is in $\rho_{n+1}(h(E))$. We conclude that $h \circ \rho_{n+1}$ maps $E$ into $X_{n} \cup \cup_{x \in X_{n} \backslash\left\{o_{n}\right\}} e_{x}(W)$. This union can be continuously retracted onto $X_{n}$ by $\rho\left(e_{x}(y)\right)=x$ for every $y \in W$. Hence $h \circ \rho_{n+1} \circ \rho$ maps $E$ continuously into $X_{n}$ such that the image contains $o_{n}$. We show that $h \circ \rho_{n+1} \circ \rho$ is constant onto $o_{n}$ (and then $h \circ \rho_{n+1}$ is also constant onto $o_{n}$ ). Let $n=0$. If $E \in \mathbb{Q}^{1} \cup \mathbb{Q}^{2}$, then it follows from 5; if $E \in\{B\} \cup \mathbb{Q}^{3}$, then it follows either from $\S I V .3$ or from $\S I V .4$. Let us suppose $n \geqslant 1$. For every $x \in$ $X_{n-1} \backslash\left\{o_{n}\right\}$, put $\mathscr{Q}_{x}=e_{x}\left(Q \backslash W \cup\left\{q_{1}, q_{2}, q_{3}\right\}\right)$ again and let $G_{x}$ be its preimage in $h \circ \rho_{n+1} \circ \rho$. Since $E \backslash G_{x}$ is always nonempty, $G_{x}$ must be empty, again by 2 . But $\left\{o_{n}\right\} \cup \cup{ }_{x} \mathscr{Q}_{x}$, where $x$ runs over $X_{n-1} \backslash\left\{o_{n}\right\}$, is a neighbourhood of $o_{n}$ in $X_{n}$. Since $E$ is connected, its image is just $\left\{o_{n}\right\}$.

7. Lemma. Let $E$ be in $\mathcal{E}$, let $h: E \rightarrow X$ be continuous and let $o_{m} \notin h(E)$ for all $m \geqslant n$. Let $l \geqslant n$ and let $h \circ \rho_{l}$ map $E$ into one $W$-branch of $X$. If either $E \in\{B\} \cup$ $\mathbb{Q}^{3}$ or $h \circ \rho_{l}$ is nonconstant, then $h \circ \rho_{l+1}$ maps $E$ into one $W$-branch.

Proof. Denote $g=h \circ \rho_{l}, \bar{g}=h \circ \rho_{l+1}$. (a) First, let us suppose that $g$ is nonconstant. Since $E$ is connected, $g(E) \subset X_{l} \backslash\left\{o_{l}\right\}$ and $\bar{g}(E) \subset X_{l+1} \backslash\left\{o_{l+1}\right\}$, by §III.4. For every $x \in X_{l} \backslash\left\{o_{l}\right\}$, denote $\mathscr{Q}_{x}=e_{x}\left(Q \backslash W \cup\left\{q_{2}, q_{3}\right\}\right), G_{x}=\bar{g}^{-1}\left(\mathcal{U}_{x}\right)$. 
Since $g$ is nonconstant, $E \backslash G_{x} \neq \varnothing$ for all $x \in X_{l} \backslash\left\{o_{l}\right\}$. If $G_{x} \neq \varnothing$, then the closure, $F$, of a component of $G_{x}$ is a nondegenerate subcontinuum of $E$ mapped by $\bar{g}$ onto a nondegenerate subcontinuum of $e_{x}(Q)$. By 4 , this is possible only when $\bar{g}(F) \subset e_{x}(W)$ or $E=A_{d}^{j}$ for some $j \in\{1,2\}, d \in D$ and $\bar{g}$ is the domain-restriction of $\xi_{k} \circ e_{x}$ for some $k \in K_{j}$. The last case is impossible because $g(E) \backslash\{x\} \neq \varnothing$. We conclude that $\bar{g}$ maps $E$ into $g(E) \cup \cup_{x \in g(E)} e_{x}(W)$. If $g(E)$ is contained in one $W$-branch, then $\bar{g}(E)$ is contained also in one $W$-branch.

(b) Let us suppose that $g$ is constant with the value $y$ and $E \in\{B\} \cup \mathbb{Q}^{3}$. If $y=o_{m}$ with $m \geqslant l$, then $\bar{g}=g$. Let us suppose that $y \in X_{l} \backslash\left\{o_{l}\right\}$. Then $\bar{g}$ maps $E$ into $e_{y}(Q)$. Thus either $\bar{g}$ is constant or $\bar{g}(E) \subset e_{y}(W)$, by 4 . Consequently, $\bar{g}$ maps $E$ into one $W$-branch.

8. Lemma. Let $A$ be in $\mathbb{Q}^{1} \cup \mathbb{Q}^{2}$ and let $h: A \rightarrow X$ be continuous. Then either $h(A)$ is contained in one $W$-branch or there exists $\gamma=(j, k, x) \in \Gamma$ such that $A=A_{d}^{j}$ for some $d \in D$ and $h$ is the domain-restriction of $\sigma_{\gamma}: A^{j} \stackrel{\xi_{k}}{\rightarrow} Q \stackrel{e_{x}}{\rightarrow} X$.

(For the definition of $\Gamma$ see 3.$)$

Proof. If $h(A)$ does not intersect the sequence $\left\{o_{n}\right\}$, then $h \circ \rho_{0}$ is constant, by 5 and §III.4. If $h(A)$ intersects $\left\{o_{n}\right\}$ and $n$ is the greatest natural number such that $o_{n} \in h(A)$, then $h \circ \rho_{n+1}$ is constant, by 6 . We conclude that there is always a natural number $m$ such that $h \circ \rho_{m}$ is constant. If $h \circ \rho_{l}$ is constant for all $l \geqslant m$, then $h$ is constant, hence $h(A)$ is in one $W$-branch. Let $m$ be the smallest natural number such that $h \circ \rho_{m}$ is nonconstant. By $5, m \geqslant 1$. Let $y$ be in $\tilde{X}_{m-1}$ such that $h \circ \rho_{m-1}$ is the constant with the value $i(y)$, where $\tilde{X}_{m-1}$ and $i: \tilde{X}_{m-1} \rightarrow X$ are as in §III.4. Then necessarily $y \in X_{m-1} \backslash\left\{o_{m-1}\right\}$ otherwise $h \circ \rho_{m}$ would be equal to the constant onto $o_{m-1}$ again. Hence $i(y)=y$ and $h \circ \rho_{m}$ maps $A$ into $e_{y}(Q)$. Since $h \circ \rho_{m}$ is supposed to be nonconstant, either it maps $A$ into $e_{y}(W)$ or it is the domain-restriction of some $\sigma_{\gamma}: A^{j} \stackrel{\xi_{k}}{\rightarrow} Q \stackrel{e_{x}}{\rightarrow} X$, where $\gamma=(j, k, x), A=A_{d}^{j}$ for some $d \in D$. In the second case, necessarily $h \circ \rho_{m}=h$ because $h(A)$ is connected. If $h \circ \rho_{m}$ maps $A$ into $e_{y}(W)$, i.e. into one $W$-branch, then by $7, h \circ \rho_{l}$ is nonconstant and maps $A$ into one $W$-branch for all $l \geqslant m$. Then $h$ maps $A$ into one $W$-branch.

9. Proposition. Let $h: Q \rightarrow X$ be a continuous mapping. Then either $h(Q)$ is in one $W$-branch or $h=e_{x}$ for some $x \in X$.

Proof. (a) For every $j \in\{1,2\}, k \in K_{j}, d \in D$, the mapping $A_{d}^{j} \hookrightarrow A^{j} \stackrel{\xi_{k}}{\rightarrow} Q \stackrel{h}{\rightarrow} X$ maps $A_{d}^{j}$ either into one $W$-branch or it is the domain-restriction of some $\xi_{\bar{k}} \circ e_{x}$, by 8. If $\left\{B_{1}, \ldots, B_{n}\right\}$ is a circle in $A^{j}$ (for the definition of the circle in $A^{j}$, see $\S$ IV.2) and $\xi_{k} \circ h$ maps $B_{1}$ into one $W$-branch, then it maps $\cup_{i=1}^{n} B_{i}$ into one $W$-branch, by the observation in 3 . We conclude that if $\xi_{k} \circ h$ maps at least one $A_{d}^{j}$ into one $W$-branch, then it maps the whole $A^{j}$ into one $W$-branch, by $\S I V .2$. Otherwise there exist $x=x(j, k) \in X$ and $\bar{k}=\bar{k}(j, k) \in K_{j}$ such that $\xi_{k} \circ h=\xi_{\bar{k}} \circ e_{x}$.

(b) Let us suppose that, for some $j \in\{1,2\}, k \in K_{j}$, the mapping $\xi_{k} \circ h$ maps $A^{j}$ into one $W$-branch. We recall that $K_{1}=Z, K_{2}=M$, where $M$ is a connected rigid relation on $Z$ (see 2). Let us suppose $j=1, k=z \in Z$. Choose $m \in M$ such that 
$\pi_{l}(m)=z$, where $\pi_{l}$ is the first or the second projection. Then necessarily $h\left(\xi_{m}\left(a^{2, l}\right)\right)$ and $h\left(\xi_{m}\left(a^{2,3}\right)\right)$ are in the same $W$-branch, consequently $\xi_{m} \circ h$ maps $A^{2}$ into one $W$-branch, by (a). Since $M$ has been chosen to be connected, $h$ maps $\cup_{m \in M} \xi_{m}\left(A^{2}\right)$ into one $W$-branch. Hence it maps every $\xi_{z}\left(a^{1,2}\right)$ and $\xi_{z}\left(a^{1,3}\right)$ in the same $W$-branch, consequently $h$ maps the whole $Q$ into one $W$-branch.

(c) Otherwise for every $j \in\{1,2\}$ and $k \in K_{j}$ there exists $\bar{k}=\bar{k}(j, k) \in K_{j}$ and $x=x(j, k) \in X$ such that $\xi_{k} \circ h=\xi_{\bar{k}} \circ e_{x}$. Since $\xi_{k}\left(a^{j, 3}\right)=\xi_{k^{\prime}}\left(a^{j^{\prime}, 3}\right)$ for all $j, j^{\prime} \in$ $\{1,2\}, k \in K_{j}, k^{\prime} \in K_{j^{\prime}}$, necessarily $x(j, k)=x\left(j^{\prime}, k^{\prime}\right)$; denote it simply by $x$. Define $f: Z \rightarrow Z$ by $f(k)=\bar{k}$. If $m=\left(z_{1}, z_{2}\right) \in M$ then $\xi_{z_{1}}\left(a^{1,2}\right)=\xi_{m}\left(a^{2,1}\right)$ and $\xi_{z_{2}}\left(a^{1,2}\right)=\xi_{m}\left(a^{2,2}\right)$. This implies that $f:(Z, M) \rightarrow(Z, M)$ is a morphism of $\mathbf{G}$. Since $M$ has been chosen to be rigid (see 2), $\bar{k}=k$ for all $k \in Z=K_{1}$. Then also $\bar{k}=k$ for all $k \in K_{2}=M$. We conclude that $h=e_{x}$.

10. Proposition. Let us suppose $(P, p)=\left(\mathfrak{T}(G), p_{G}\right)$, let $g: \Re\left(G^{\prime}\right) \rightarrow X$ be a continuous mapping. Then $g\left(\mathfrak{N}\left(G^{\prime}\right)\right)$ is contained either in $\mathscr{N}(G)$ or in one $W$ branch of $X$.

Proof. (a) First, let us consider a continuous mapping $h: A_{d}^{3} \rightarrow X, d \in D$. If either $h\left(A_{d}^{3}\right)$ intersect $\left\{o_{n}\right\}$ or $h \circ \rho_{0}$ is constant, then $h\left(A_{d}^{3}\right)$ is contained in one $W$-branch, by 6 and 7. Let us suppose that $h \circ \rho_{0}$ is nonconstant. By $\S I I I .4$, it maps $A_{d}^{3}$ into $\mathfrak{R}(G) \backslash\left\{p_{G}\right\}$. By $\S \mathrm{IV} .3, h \circ \rho_{0}$ is the domain-restriction of $e_{r}: A^{3} \rightarrow \mathfrak{R}(G)$ for some $r \in R$, where $G=(S, R)$. Since $h\left(A_{d}^{3}\right)$ is connected, $h \circ \rho_{0}=h$, i.e., $h$ is the domain-restriction of $e_{r}$.

(b) Now, let $h: A^{3} \rightarrow X$ be continuous. If $h$ maps $A_{d}^{3}$ into one $W$-branch for some $d \in D$, then it maps the whole $A^{3}$ into one $W$-branch. For, if $\left\{B_{1}, \ldots, B_{n}\right\}$ is a circle in $A^{3}$ and $h$ maps $B_{1}$ into one $W$-branch, it maps $\cup_{i=1}^{n} B_{i}$ into one $W$-branch, by (a) and 3, hence it maps $A^{3}$ into one $W$-branch. Otherwise $h\left(A^{3}\right) \subset$ $\Re(G)$ and $h$ is nonconstant, hence $h=e_{r}$ for some $r \in R$, by $\S I V .3$.

(c) If $g$ : $\Re\left(G^{\prime}\right) \rightarrow X$ is continuous, $G^{\prime}=\left(S^{\prime}, R^{\prime}\right)$, then for every $r \in R^{\prime}, e_{r} \circ g$ : $A^{3} \rightarrow X$ is either $e_{\bar{r}}$ for some $\bar{r} \in R$ or it maps $A^{3}$ into one $W$-branch. If $e_{r} \circ g$ maps $A^{3}$ into one $W$-branch then it maps the whole $\mathfrak{N}\left(G^{\prime}\right)$ into one $W$-branch because $G^{\prime}$ is connected. Otherwise $g\left(\mathfrak{N}\left(G^{\prime}\right)\right) \subset \mathfrak{R}(G)$.

11. We recall that $B$ is a nondegenerate subcontinuum of a Cook continuum $e$ disjoint with all members of $\mathbb{Q}^{1} \cup \mathbb{Q}^{2} \cup \mathbb{Q}^{3}$ (see the beginning of $\S \mathrm{V}$ ). Let $S^{\prime}$ be a set. Since $B^{S^{\prime}}$ is compact, $h\left(B^{S^{\prime}}\right)$ contains only a finite number of members of the sequence $\left\{o_{n}\right\}$ for every continuous mapping $h: B^{S^{\prime}} \rightarrow X$. Let $h\left(B^{S^{\prime}}\right)$ really intersect $\left\{o_{n}\right\}$ and let $n$ be the greatest natural number such that $o_{n} \in h\left(B^{S^{\prime}}\right)$.

LEMMA. $h \circ \rho_{n+1}: B^{S^{\prime}} \rightarrow X$ is constant onto $o_{n}$.

Proof. (a) If card $S^{\prime}=1$, then it follows from 6.

(b) Let $S^{\prime}$ be arbitrary, nonempty. Choose $\varphi_{0} \in B^{S^{\prime}}$ with $h\left(\varphi_{0}\right)=o_{n}$. For every $s \in S^{\prime}$ denote by $\eta_{s}: B \rightarrow B^{S^{\prime}}$ the embedding sending any $x \in B$ to $\varphi$ such that $\varphi(s)=x, \varphi(t)=\varphi_{0}(t)$ for all $t \in S^{\prime} \backslash\{s\}$. Then $n$ is the greatest natural number such that $o_{n} \in h\left(\eta_{s}(B)\right)$; hence $\eta_{s} \circ h \circ \rho_{n+1}$ is constant onto $o_{n}$, by (a). We conclude that $h \circ \rho_{n+1}$ maps onto $o_{n}$ every $\varphi \in B^{S^{\prime}}$ which differs from $\varphi_{0}$ for at 
most one $s \in S$. Since $\left(h \circ \rho_{n+1}\right) \circ \rho_{n+1}=h \circ \rho_{n+1}$, we can proceed by induction. We obtain that $h \circ \rho_{n+1}$ maps onto $o_{n}$ every $\varphi \in B^{S^{\prime}}$ which differs from $\varphi_{0}$ for at most a finite number of $s \in S^{\prime}$. Since this set is dense in $B^{S^{\prime}}, h \circ \rho_{n+1}$ maps $B^{S^{\prime}}$ onto $o_{n}$.

12. LemmA. Let $h: B^{S^{\prime}} \rightarrow X$ be a continuous mapping, let $o_{m} \notin h\left(B^{S^{\prime}}\right)$ for all $m \geqslant n$. Let $l \geqslant n$. If $h \circ \rho_{l}$ maps $B^{S^{\prime}}$ into one $W$-branch, then $h \circ \rho_{l+1}$ maps $B^{S^{\prime}}$ also in one $W$-branch.

Proof. (a) If card $S^{\prime}=1$, then it follows from 7.

(b) Let $S^{\prime}$ be arbitrary, nonempty. Choose $\varphi_{0} \in B^{S^{\prime}}$ and define $\eta_{s}: B \rightarrow B^{S^{\prime}}$ as in the previous proof. Since $\eta_{s} \circ h \circ \rho_{l}$ maps $B$ into one $W$-branch, $\eta_{s} \circ h \circ \rho_{l+1}$ maps it into one $W$-branch, by (a). We conclude that $h \circ \rho_{l+1}$ maps every $\varphi \in B^{S^{\prime}}$, which differs from $\varphi_{0}$ for at most one $s \in S$, into the same $W$-branch as $\varphi_{0}$. Since $h \circ \rho_{l}$ maps $B^{S^{\prime}}$ into one $W$-branch, we can proceed by induction. Thus, $h \circ \rho_{l+1}$ maps the whole $B^{S^{\prime}}$ into one $W$-branch.

13. Proposition. Let $(P, p)=\left(\mathscr{P}(\omega), p_{\omega}\right)$, let $g: \mathcal{P}\left(\omega^{\prime}\right) \rightarrow X$ be a continuous mapping. Then $g\left(\mathcal{P}\left(\omega^{\prime}\right)\right)$ is contained either in $\mathscr{P}(\omega)$ or in one W-branch.

Proof. Denote $\omega=(S, \mathcal{T})$. (a) First, let us consider a continuous mapping $h$ : $A_{d}^{3} \rightarrow X, d \in D$. If $h\left(A_{d}^{3}\right)$ intersects $\left\{o_{n}\right\}$ and $n$ is the greatest $n$ such that $o_{n} \in h\left(A_{d}^{3}\right)$, then $h \circ \rho_{n+1}$ is constant, by 6. Otherwise consider $h \circ \rho_{0}$. This is either constant or maps $A_{d}^{3}$ into $X_{0} \backslash\left\{o_{0}\right\}$. If $h \circ \rho_{l}$ is constant for some $l$, then $h$ maps $A_{d}^{3}$ into one $W$-branch, by 7 . Otherwise $h \circ \rho_{0}$ maps $A_{d}^{3}$ into $\mathscr{P}(\omega) \backslash\left\{p_{\omega}\right\}$. Then, by \$IV.4, there exists $T \in \mathcal{T}$, such that $h \circ \rho_{0}$ sends every $a \in A_{d}^{3}$ to $(a, T)$. Then $h \circ \rho_{0}=h$ because $h(A)$ is connected.

(b) Now, let $h: A^{3} \rightarrow X$ be a continuous mapping. By (a) it maps every $A_{d}^{3}$ either in one $W$-branch or onto $A_{d}^{3} \times\{T(d)\}$ for some $T(d) \in \mathcal{T}$ by the rule $a \leadsto$ $(a, T(d))$. If it maps some $A_{d}^{3}$ into one $W$-branch, then it maps the whole $A^{3}$ into one $W$-branch (the proof using circles in $A^{3}$ is quite analogous to $10(\mathrm{~b})$ ). Otherwise all the $T(d)$ 's are equal to each other, i.e. $h$ maps $A^{3}$ into $\mathcal{P}(\omega)$ by the rule $a \leadsto(a, T)$.

(c) Let $g: \mathcal{P}\left(\omega^{\prime}\right) \rightarrow X$ be a continuous mapping. Denote $\omega^{\prime}=\left(S^{\prime}, \mathcal{T}\right)$. Since $B^{S^{\prime}}$ is compact, $g\left(B^{S^{\prime}}\right)$ contains only a finite number of members of the sequence $\left\{o_{n}\right\}$. If it really intersects it, then $g \circ \rho_{n+1}$ is constant on $B^{S^{\prime}}$ for suitable $n$. If $g \circ \rho_{l}$ is constant on $B^{S^{\prime}}$ for some $l$, then $g$ maps $B^{S^{\prime}}$ into one $W$-branch, by 12 . Then, by (b), $g$ maps every $A^{3} \times\{T\}$ into one $W$-branch, hence $g$ maps the whole $\mathcal{P}\left(\omega^{\prime}\right)$ into one $W$-branch. If $g\left(B^{S^{\prime}}\right)$ does not intersect $\left\{o_{n}\right\}$, and $g \circ \rho_{0}$ is not constant on $B^{S^{\prime}}$, it maps $B^{S^{\prime}}$ into $\mathscr{P}(\omega) \backslash\left\{p_{\omega}\right\}$. If, for some $T \in \mathcal{T}^{\prime}, g$ maps $A^{3} \times\{T\}$ into one $W$-branch, then $g\left(\chi_{T}\right)=g\left(b^{S^{\prime}}\right)$, both being in one $W$-branch and also in $\mathscr{P}(\omega) \backslash\left\{p_{\omega}\right\}$, where $\chi_{T}$ and $b^{S^{\prime}}$ are as in $\S I V .4$. Then, by $\S I V .4, g$ is constant on $B^{S^{\prime}}$, which is a contradiction. Hence, $g$ maps every $A^{3} \times\{T\}$ into $\mathscr{P}(\omega) \backslash\left\{p_{\omega}\right\}$, consequently $g\left(\mathcal{P}\left(\omega^{\prime}\right)\right) \subset \mathcal{P}(\omega)$.

14. Now, we finish the proofs of the main theorems. Denote by $L$ the category of all paracompact or all normal or all completely regular or all regular or all 
Hausdorff or all topological spaces. The following assertion follows immediately from 9, 10, 11, §§IV and III.10.

Let spaces $Y, V$ be given. Form $W, w$ and $T$ as in $\S$ III.5 and \$III.6. Let $\mathcal{E}$ be a countable collection of nondegenerate subcontinua of a Cook continuum $\mathcal{C}$ and let $H$ be a space with two distinguished points $h_{1}, h_{2}$ such that (1), and (2) from 1 are fulfilled. Let $\mathbf{K}$ be the category of all spaces $X$ containing $V$ many times and such that every continuous mapping $f: X \rightarrow Y$ is constant. If $V$ and $H$ are in $\mathbf{L}$, then $\mathbf{K} \cap \mathbf{L}$ is an almost universal category. If $V$ and $H$ are in the category $\mathbf{M}$ of all metrizable spaces, then $\mathbf{K} \cap \mathbf{M}$ is an almost $s$-universal category (and, under (M), it is universal).

To prove Theorem 1, we choose $H$ to be a totally disconnected regular space such that no continuous mapping of $H$ into any space of the cardinality card $Y+$ card $T$ distinguishes $h_{1}$ from $h_{2}$ (such a space is constructed in [6] or [2]). Since $H$ is totally disconnected, any continuous mapping of any $E \in \mathcal{E}$ into $H$ is constant. To prove Theorem 2 and Theorem 3, it is sufficient to choose $Y$ to be a one-point space and $H$ to be a nondegenerate subcontinuum of $\mathcal{C}$ disjoint with all $E \in \mathcal{E} ; h_{1}$ and $h_{2}$ can be arbitrary distinct points of $H$. Let us note that, for the proofs of Theorem 2 and Theorem 3, the whole construction can be essentially simplified.

15. REMARK. The main theorems were announced in [16], where the category of all connected compact Hausdorff spaces, containing a given totally disconnected compact Hausdorff space many times, is also announced to be $s$-universal (and, under (M), universal). Since the present paper is rather long and the proof of the assertion about compact Hausdorff spaces requires quite distinct construction, it will appear elsewhere.

ACKNOWLEDGEMENT. I am indebted to my colleague Jana Bočková, who has carefully read the whole manuscript and made several valuable comments (but she is not responsible for inaccuracies in my writing).

\section{REFERENCES}

1. H. Cook, Continua which admit only the identity mapping onto non-degenerate subcontinua, Fund. Math. 60 (1967), 241-249.

2. T. E. Gantner, A regular space on which every continuous real-valued function is constant, Amer. Math. Monthly 78 (1971), 52-53.

3. J. de Groot, Groups represented by homeomorphisms groups. I, Math. Ann. 138 (1959), 80-102.

4. Z. Hedrlin, Non-constant continuous transformations form any semigroup with a unity, Nieuw Arch. Wisk. 14 (1966), 230-236.

5. Z. Hedrlin and J. Lambek, How comprehensive is the category of semigroups, J. Algebra 11 (1969), $195-212$.

6. H. Herrlich, Wann sind alle stetigen Abbildungen in Y konstant? Math. Z. 90 (1965), 152-154.

7. __ Topologische reflexionen und coreflexionen, Lecture Notes in Math., vol. 78, SpringerVerlag, Berlin and New York, 1968.

8. E. Hewitt, On two problems of Urysohn, Ann. of Math. (2) 47 (1946), 503-509.

9. J. R. Isbell, Subobjects, adequacy, completeness and categories of algebras, Rozprawy Mat. 36 (1964).

10. V. Koubek, Each concrete category has a representation by $T_{2}$-paracompact topological spaces, Comment. Math. Univ. Carolinae 15 (1974), 655-663.

11. L. Kučra, Lectures on the theory of categories, Charles University, 1970 (preprint). (Czech)

12. J. Novák, Regular space on which every continuous function is constant, Casopis Pèst. Mat. Fys. 73 (1948), 58-68. 
13. A. Pultr and V. Trnková, Combinatorial, algebraic and topological representations of groups, semigroups and categories, North Holland, Amsterdam, 1980; Czechoslovak Publ. House, Academia, Prague, 1980.

14. V. Trnková, Non-constant continuous mappings of metric or compact Hausdorff spaces, Comment. Math. Univ. Carolinae 13 (1972), 283-295.

15. All small categories are representable by continuous nonconstant mappings of bicompacta, Soviet. Math. Dokl. 17 (1976), 1403-1406.

16. , Categorial aspects are useful for topology, Proceedings of the IVth Prague Topological Symposium, Lecture Notes in Math., vol. 609, Springer-Verlag, Berlin and New York, pp. 211-215.

17. P. Vopènka, A. Pultr and Z. Hedrlin, A rigid relation exists on any set, Comment. Math. Univ. Carolinae 6 (1965), 149-155.

Mathematical Institute, Charles University, Sokolovska 83, 18600 Praha, Czechoslovakia 